\title{
On-Orbit Measurement of Next Generation Space Solar Cell Technology on the International Space Station
}

David S. Wolford ${ }^{1}$, Matthew G. Myers ${ }^{1}$, Norman F. Prokop ${ }^{1}$, Michael J. Krasowski ${ }^{1}$, David S. Parker ${ }^{2}$, Justin C. Cassidy², William E. Davies ${ }^{3}$, Janelle O. Vorreiter ${ }^{4}$, Michael F. Piszczor ${ }^{1}$ and Jeremiah S. McNatt ${ }^{1}$

${ }^{1}$ NASA Glenn Research Center, Cleveland, Ohio 44135

2'Goddard Space Flight Center, Code 408/ Lockheed Martin, Greenbelt MD 20771 ${ }^{3}$ Goddard Space Flight Center, Code 408 /Jackson and Tull, Greenbelt MD 20771 ${ }^{4}$ Goddard Space Flight Center, Code 408 / ATK Space Systems, Greenbelt MD 20771 
- Introduction

- Background

- Experiment Description

- Mission Description

- Summary

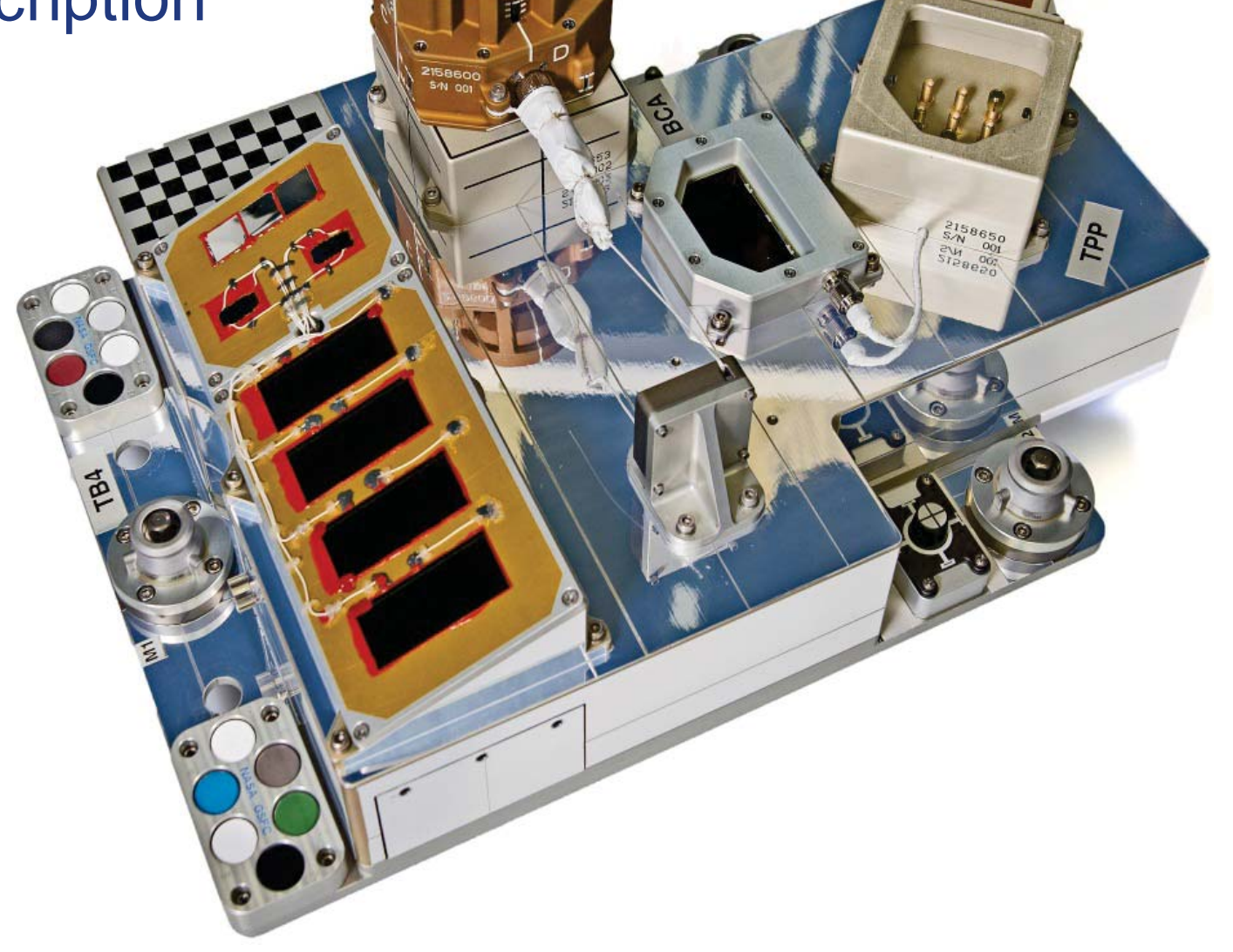

Robotic Refueling Mission’s (RRM) Task Board 4 (TB4) 
$\underline{\text { Introduction }}$

- A collaboration between Goddard Space Flight Center (GSFC) and Glenn Research Center (GRC)

- Enabled by RRM, the Solar Cell Experiment (SCE) will fly advanced solar cells from 4 industry and government partners.

- SCE leverages primary experiment (RRM) to provide an additional opportunity for orbital science objectives.

- Orbital air-mass-zero (AM0) measurements are an important part of a balanced photovoltaic (PV) measurement program

- SCE will be self-contained.

- Self powered (PV)

- Internal memory

- Experiment will be de-orbited for evaluation and recovery of data 


\section{Background}

- Primary AM0 measurements do not depend on laboratory solar simulators which use reference standards for calibration.

- High value, high cost, occasional availability

- Materials International Space Station Experiment (MISSE) has been the main method for recent advanced PV measurements on ISS.

- 8 missions since 2001

- Participants

- GRC, LEX: P.I., PV measurement, PV partner co-ordination

- GRC, LCP, Mobile And Remote Sensing Lab: Measurement electronics and controls

- GSFC, Satellite Servicing Capabilities Office (SSCO): Mechanical design and fabrication, wiring harness design and fabrication, qualification testing, hardware integration.

- 11 next-generation Inverted Metamorphic Multi-junction (IMM) cells and component cells on board 


\section{Experiment Description}

- Active Measurement area on back of TB4

- Contains 10 test cells and 4 power cells

- 3 industry partners

- Advanced IMM cells

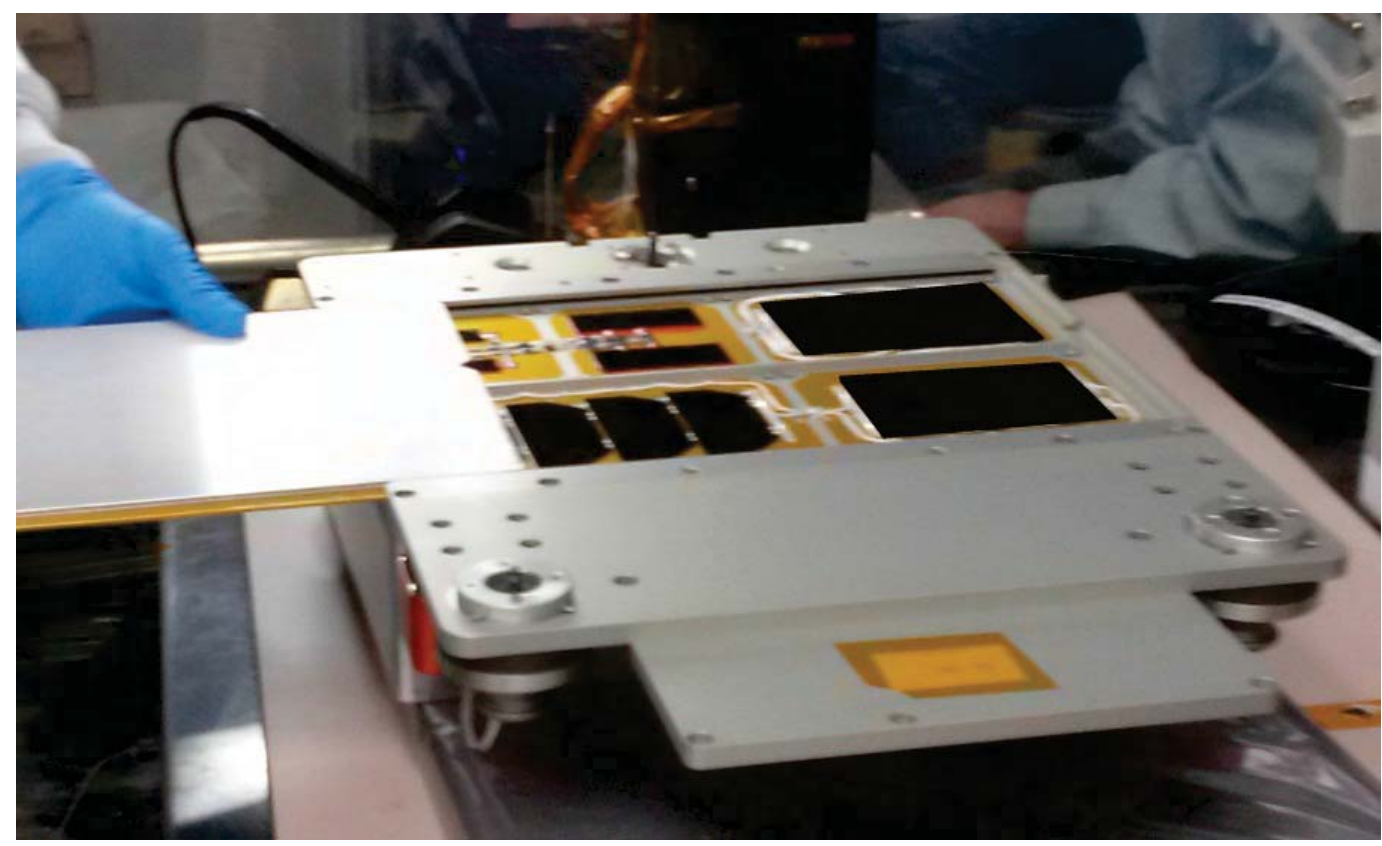

Active measurement area on back of TB4. (GRC, edited) 


\section{Experiment Description}

- Passive Measurement area on front of TB4

- Contains 6 test cells 3 industry partners

- Advanced IMM and Microsystems-Enabled PV cells

- 3 PV adhesive test samples

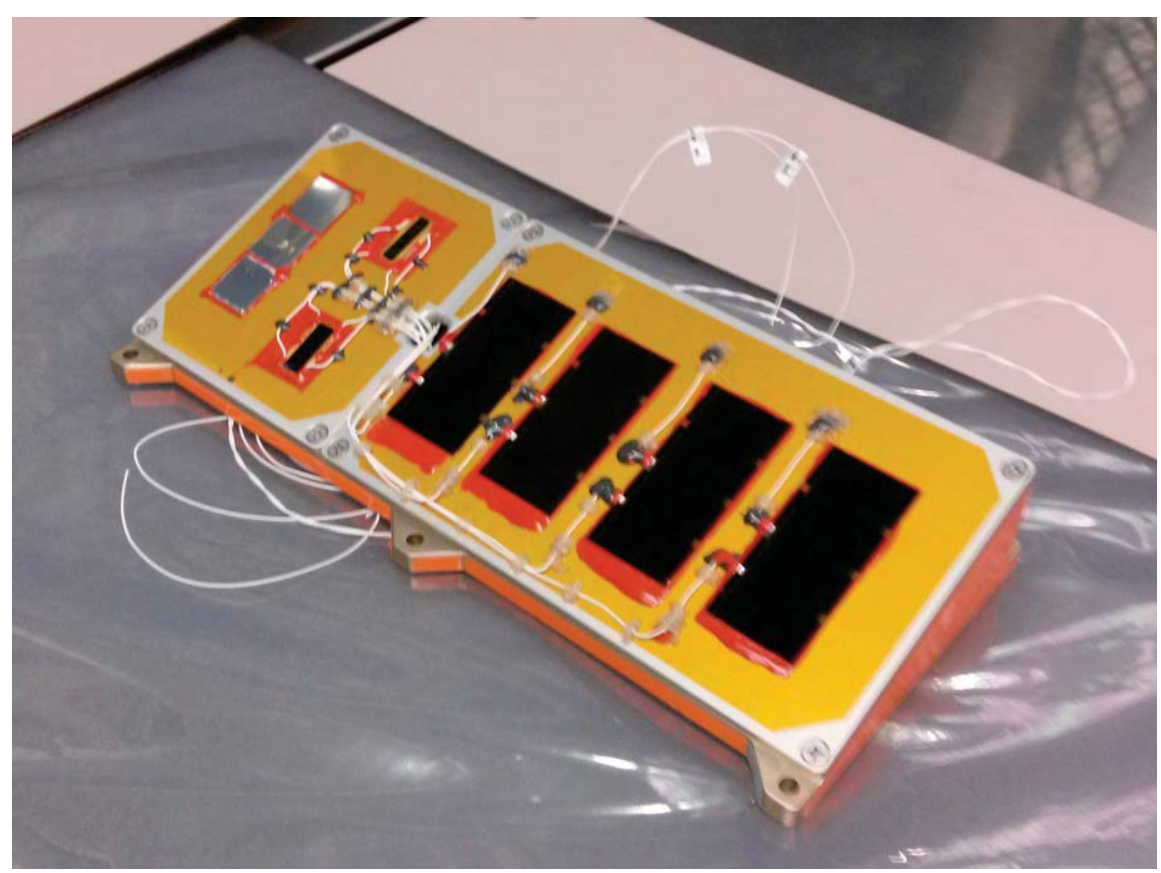

Passive test sample area on front of TB4. (GRC) 


\section{Experiment Description}

- Measurement Electronics

- 2 Printed Wiring Assemblies (PWA) each with 4 measurement channels and flash memory

- Flight heritage from MISSE

- Processor "wakes up" when sufficient power is being generated by the 4 cell power string

- Power must be maintained for 1 minute before data is collected

- Current and voltage will be recorded at 128 intervals

- One minute pause between each 4 channel scan

- Temperature for each channel recorded at the beginning and end of scan

- Memory capacity for 34 hours of continuous operation

- TB4 can be pointed off-sun to conserve memory

- Flash memory will not over-write 


\section{Experiment Description}

\section{- Location}

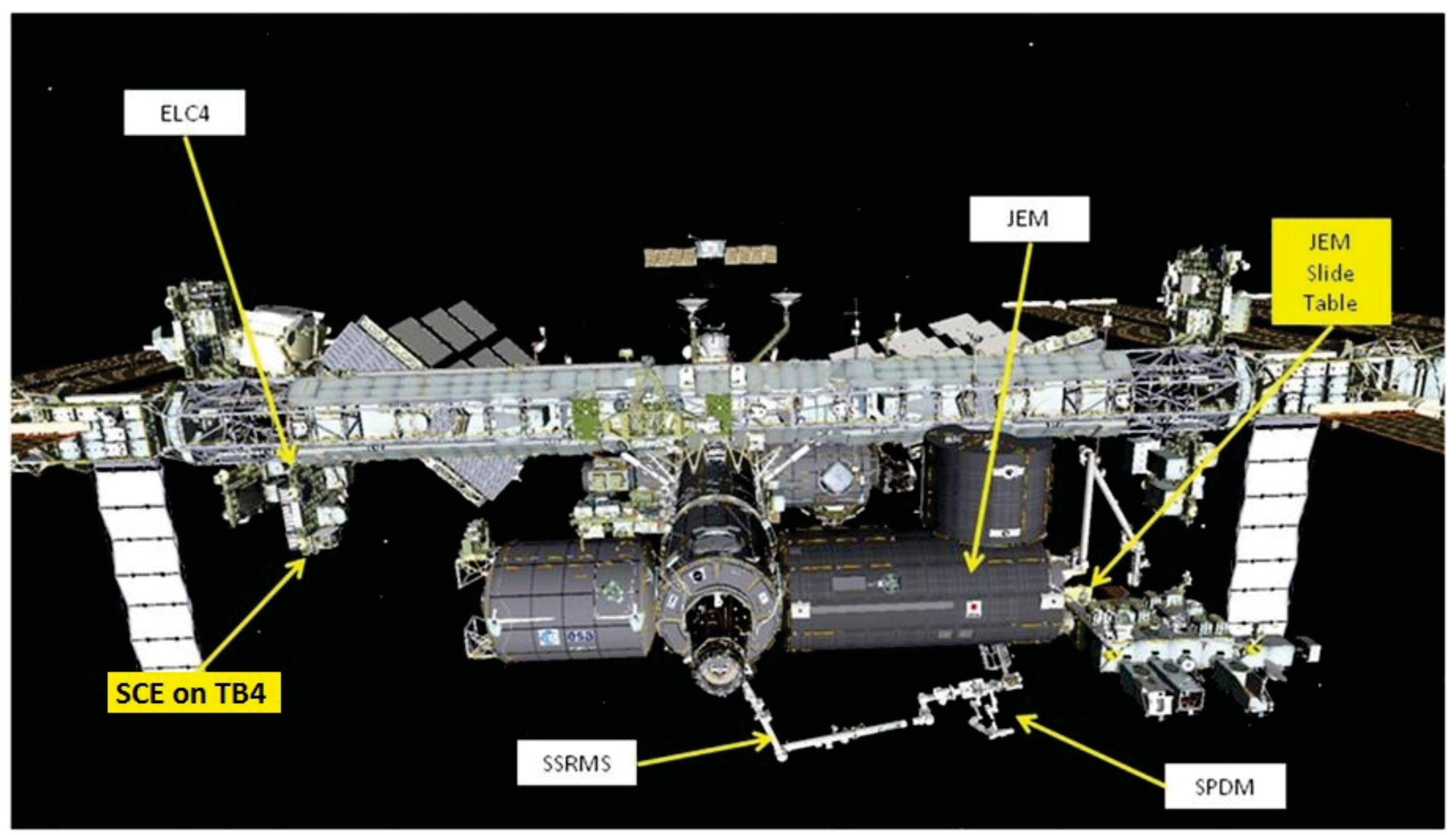

Location of SCE indicated at lower left, view from ram direction (GSFC) 


\section{Mission Description}

- The hardware was launched on the European Space Agency's Automated Transfer Vehicle "Georges Lemaitre" (ATV-5) on July 29, 2014

- Launched at Europe's Spaceport in French Guiana

- Will be moved through JEM air-lock in December 2014

- TB4 will be directed at the sun by SPDM by robotic ground operators guided by ISS and SPDM camera feeds

- Task board 4 will then be placed on the RRM experiment located on the Express Logistics Carrier 4 (ELC4)

- Additional set of measurements can be accomplished during the mission by retrieving TB4 and repeating the exposure routine

- A last set of measurements will be made when TB4 is retrieved at the end of the 18 month mission

- Data and flight samples will returned to GRC for measurement and analysis before being returned to the company of origin 
Mission Description

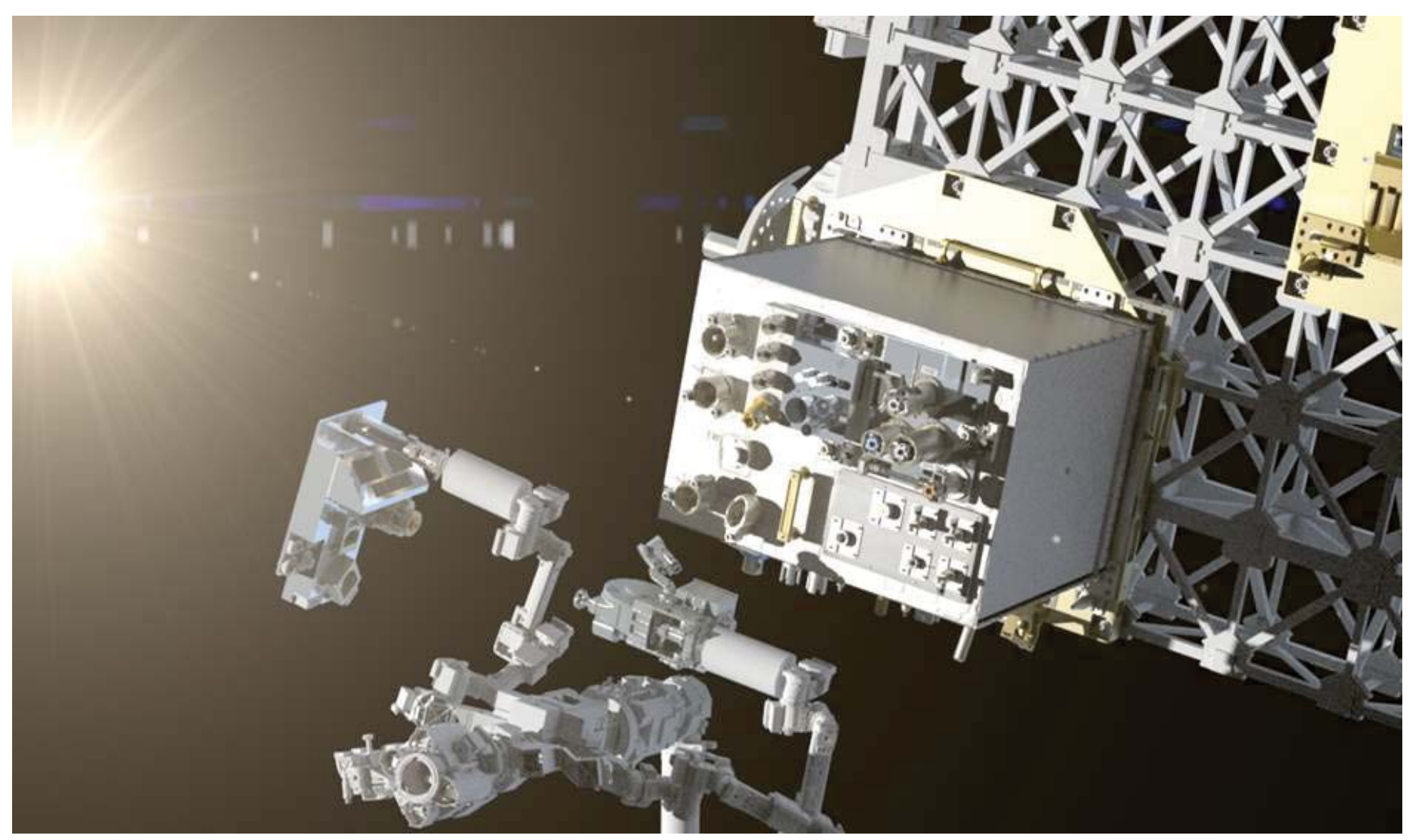

Graphic depicting robotic sun-pointing during the mission (GSFC) 
Summary

- In collaboration with GSFC, GRC has a solar cell experiment on board the ISS. First set of active measurements to occur in December 2014.

- SCE takes advantage of access to space via GSFC's RRM TB4.

- Ten advanced AMO solar cells, from three industry partners, will have their electrical characteristics measured in space.

- Nine samples, from three industry and government partners, will be exposed for eighteen months to the LEO environment.

- The data from this experiment will provide valuable performance metrics of new technology to manufacturers. NASA will benefit from the opportunity to verify its laboratory measurement accuracy and observe PV technological progress.

- This collaboration fosters a mutually beneficial relationship NASA has with the space PV community

wolford@nasa.gov 